\title{
5 eXPectativas face aO Álcool e competênCIAS de AUTORREGULAÇÃO DOS ADOLESCENTES
}

\author{
| Manuela Ferreira ${ }^{1}$; Gonçalo Valente ${ }^{2}$; Lidia Cabral ${ }^{3}$; João Duarte ${ }^{4} \mid$
}

\section{RESUMO}

CONTEXTO: O consumo de álcool na adolescência está associado a comportamentos de risco, baixo rendimento académico, dificuldades de aprendizagem, baixo nível de desenvolvimento de competências sociais e emocionais. Pode causar alterações no desenvolvimento da personalidade e prejudicar funções como memória e atenção. O desenvolvimento de comportamentos auto-regulados permite ao adolescente o controlo das necessidades mais imediatas (controlo de impulsos) assim como a mobilização de pensamentos, sentimentos e comportamentos para objetivos de saúde a longo prazo. OBJETIVO(S): Analisar a relação entre as expectativas face ao álcool e as competências de auto-regulação em adolescentes.

METODOLOGIA: Recorreu-se a um modelo de investigação quantitativo, transversal, analítico, descritivo e correlacional. Participaram 971 estudantes do ensino secundário público e cooperativo. O protocolo de avaliação inclui o questionário sociodemográfico, a Escala de Envolvimento com o Álcool para Adolescentes de Mayer \& Filstead (1979) adaptada por Fonte e Alves (1999), o Questionário Reduzido de Auto-regulação (Carey, Neal \& Collins, 2004 adaptado por Castillo \& Dias, 2009) e o Questionário de Expectativas face ao Álcool para Adolescentes (Pilatti, Godoy \& Brussino, 2010). RESULTADOS: Os estudantes com idades compreendidas entre os 14-21 anos, na sua maioria rapazes (50,80\%), com idade igual ou inferior a 16 anos (43,40\%), residentes em meio rural (66,40\%), em coabitação com os pais (77,30\%) e inseridos em agregados familiares com um rendimento médio mensal médio - alto ou alto (56,70\%). Revelaram-se bebedores habituais sem problemas (75,30\%) e com elevadas expectativas face ao álcool (45,10\%). A auto-regulação foi influenciada pelo envolvimento com o álcool e pelas expectativas face ao álcool.

CONCLUSÃO: O desenvolvimento de competências de auto-regulação revela-se um investimento em saúde uma vez que, o adolescente com um comportamento auto-regulado assume estilos de vida mais saudáveis, revelando um menor envolvimento com o álcool.

PALAVRAS-CHAVE: Adolescência; Álcool; Auto-regulação; Expectativas face ao álcool

\section{RESUMEN}

"Expectativas con respecto a habilidades de alcohol y de autorregulación de los adolescentes"

CONTEXTO: El consumo de alcohol en la adolescencia se asocia con conductas de riesgo, bajo rendimiento académico, problemas de aprendizaje, bajo nivel de desarrollo de las habilidades sociales y emocionales. Puede causar cambios en el desarrollo de la personalidad y deteriorar las funciones tales como la memoria y la atención. El desarrollo de la conducta autorregulado permite adolescentes de control de las necesidades más inmediatas (control de impulsos), así como la movilización de los pensamientos, sentimientos y comportamientos de los objetivos de salud a largo plazo.

OBJETIVO: Analizar la relación entre las expectativas en relación con el alcohol y las habilidades de autorregulación en los adolescentes

METODOLOGÍA: Recurrido a un modelo de investigación cuantitativa, transversal, analítico, descriptivo y correlacional. Con la asistencia de 971 estudiantes de las escuelas públicas y de cooperación. El protocolo de evaluación incluye el cuestionario sociodemográfico, la escala de implicación con alcohol a los adolescentes Mayer y Filstead (1979) adaptada por Fonte y Alves (1999), la Reducción de Autorregulación Cuestionario (Carey, Neal y Collins, 2004 adaptados por Castillo y Dias, 2009) y el Cuestionario de Expectativas en contra del alcohol a los adolescentes (Pilatti, Godoy y Brussino, 2010).

RESULTADOS: Los estudiantes mayores de 14 - 21 años, en su mayoría varones $(50,80 \%)$, menores de 16 años $(43,40 \%)$, que viven en las zonas rurales $(66,40 \%)$ en vivir con los padres $(77,30 \%)$ y se inserta en los hogares con un ingreso promedio mensual medio - alto o alto (56,70\%). Demostraron bebedores habituales y sin problemas $(75,30 \%)$ y con altas expectativas sobre el alcohol $(45,10 \%)$. La autorregulación se vio influenciado por la implicación con el alcohol y las expectativas con respecto al alcohol.

CONCLUSIÓN: El desarrollo de habilidades de autorregulación ha demostrado ser una inversión en salud ya que el adolescente con un comportamiento autorregulado asume saludable estilo de vida, revelando una menor participación con el alcohol

\section{DESCRIPTORES: Adolescencia; Alcohol; Autorregulación; Expectativas en relación con el alcohol}

\section{ABSTRACT \\ "Expectations regarding alcohol and self-regulation skills of adolescents"”}

BACKGROUND: Alcohol consumption during adolescence is associated with risky behaviors, low academic performance, learning disabilities, low level of social and emotional skills development. May cause changes in personality development and impair functions such as memory and attention. The development of self-regulated behavior allows adolescents to control their most immediate needs (impulse control) as well as to mobilize thoughts, feelings and behaviors for long-term health goals.

OBJECTIVES: To analyze the relationship between expectations towards alcohol consumption and self-regulatory skills in adolescents.

METHODS: We resorted to a quantitative, cross-sectional, analytical, descriptive and correlational research model. 971 high school students from public and cooperative schools participated in the study. The evaluation protocol includes the sociodemographic questionnaire, the Scale Teens' Involvement with Alcohol of Mayer \& Filstead (1979) adapted by Fonte \& Alves (1999), the Reduced Questionnaire of Self-regulation (Carey, Neal \& Collins, 2004 adapted by Castillo \& Dias, 2009) and the Questionnaire about expectations towards Alcohol to Adolescents (Pilatti, Godoy \& Brussino, 2010).

RESULTS: Students aged 14 - 21 years, mostly boys (50.80\%), aged under $16(43.40 \%)$, living in rural areas (66.40\%) cohabiting with parents $(77.30 \%)$ and inserted in households with an average-high or high monthly income (56.70\%). They proved to be habitual drinkers without problems $(75.30 \%)$ and with high expectations regarding alcohol (45.10\%). Self-regulation was influenced by the involvement with alcohol and the expectations regarding alcohol.

CONCLUSION: The development of self-regulatory skills proves to be a health investment. Teenagers with self-regulating behaviors assume healthier lifestyles revealing less involvement with alcohol.

\section{KEYWORDS: Adolescence; Alcohol; Self-regulation; Expecta- tions towards alcohol}

Submetido em 25-01-2016 Aceite em 30-03-2016

1 Doutora; Professora Coordenadora no Instituto Politécnico de Viseu, Escola Superior de Saúde, CI\&DETS, Viseu, Portugal, mmcferreira@gmail.com 2 Mestre; Enfermeiro no Centro Hospitalar e Universitário de Coimbra, 3000-075 Coimbra, Portugal, goncalo1043@hotmail.com

3 Doutora; Professora Coordenadora no Instituto Politécnico de Viseu, Escola Superior de Saúde, CI\&DETS, 3504-510 Viseu, Portugal, lcabral@essv.ipv.pt 4 Doutor; Professor Coordenador no Instituto Politécnico de Viseu, Escola Superior de Saúde, CI\&DETS, 3504-510 Viseu, Portugal, duarte.johnny@gmail.com

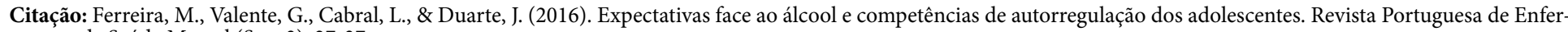
magem de Saúde Mental (Spe. 3), 27-37. 


\section{INTRODUÇÃO}

O uso de álcool na adolescência é um factor de preditor de problemas de saúde na idade adulta, além de aumentar significativamente o risco de se tornar num consumidor excessivo ao longo da vida (Strauch, Pinheiro, Silva e Horta, 2009). É manifestamente bem tolerado pela sociedade portuguesa, não sendo entendido como uma droga e por isso de fácil acesso a menores de 16 anos, nos diversos estabelecimentos (Tavares, Bonito e Oliveira, 2011). Para estes autores, está associado a determinados mitos sobre os efeitos do álcool no organismo, que dificultam a mudança de mentalidade e de comportamentos. O fenómeno do alcoolismo entre os adolescentes inicia-se cada vez mais precocemente (Sousa et al., 2008), influenciando a saúde e estilos de vida. Assim, a auto-regulação é um processo em que o indivíduo assume um papel activo na construção do seu destino (Ridder \& Wit, 2006). Ambientes que promovem o auto-controlo (caracterizados pela monitorização parental, regras claramente definidas e recompensas parentais adequadas a comportamentos positivos) surgem associadas a um menor risco de uso e dependência de álcool (Hull \& Slone, 2004). A percepção da incapacidade de controlar a ingestão de álcool tem sido usada para prever futuros consumos, particularmente em indivíduos que vivenciaram situações de dependência (Hull \& Slone, 2004). No estudo desenvolvido por Neal \& Carey (2005), os autores relatam que os estudantes universitários "consumidores pesados" de álcool obtiveram menores pontuações na avaliação geral do auto-controlo do que as pontuações obtidas pelos consumidores moderados, quando comparados com os abstinentes. Os estudos de Brown, Miller \& Lawendowski (1999), Neal \& Carey (2005) e de Hustad, Carey K., Carey M., \& Maisto (2009) concluem que a auto-regulação foi correlacionada negativamente com a impulsividade em envolver-se em múltiplos comportamentos de risco e é preditiva tanto do consumo de álcool como das consequências relacionadas com o consumo do mesmo. No estudo realizado por Pilatti et al., (2010), quando confrontadas as expectativas face ao álcool com os padrões de consumo foram encontradas diferenças significativas na antecipação das expectativas em função dos padrões de consumo, tanto para as expectativas positivas como para as negativas. Especificamente, os adolescentes com padrões de consumo excessivo e de risco apresentam maiores expectativas positivas que os adolescentes das restantes categorias de consumo. Por outro lado, os abstinentes apresentam maiores expectativas negativas.
Os autores verificaram ainda que aqueles adolescentes com um padrão de consumo excessivo e de risco mantêm mais expectativas sobre um aumento da sociabilidade que os restantes.

\section{METODOLOGIA}

Trata-se de um estudo quantitativo, descritivo/correlacional, analítico e transversal. A amostra foi constituída por 971 estudantes do ensino secundário (público e cooperativo) a frequentarem o $10^{\circ}, 11^{\circ}$ e $12^{\circ}$ ano de escolaridade, com ambos os sexos representados, sendo no entanto, maior a representatividade dos rapazes $(50,80 \%)$. Relativamente à idade, a mesma oscilou entre os 14 e os 21 anos, com uma média de 16,80 anos, sendo o grupo mais representativo o dos estudantes com idades compreendidas entre os $14 \mathrm{e}$ os 16 anos. Foi aplicado um questionário de caracterização sociodemográfica, a Escala de Envolvimento com o Álcool para Adolescentes de Mayer \& Filstead (1979) adaptada por Fonte e Alves (1999), o Questionário Reduzido de Auto-regulação (Carey, Neal \& Collins, 2004 adaptado por Castillo \& Dias, 2009) e o Questionário de Expectativas face ao Álcool para Adolescentes (Pilatti et al., 2010).

\section{RESULTADOS}

A maioria dos estudantes da amostra reside em meio rural $(66,40 \%)$ e coabitava com os pais $(77,30 \%)$, que são maioritariamente, casados ou viviam em união de facto $(84,60 \%)$. Pai e mãe detêm na sua maioria, o $2^{\circ} / 3^{\circ}$ ciclo $(45,30 \%$ e $44,50 \%$, respectivamente). A maioria dos estudantes classificou o rendimento médio do seu agregado familiar como sendo médio/alto ou alto $(56,70 \%)$. A maioria dos estudantes $(69,00 \%)$ usufrui para gastos pessoais um montante igual ou inferior a 10 euros; frequentava o $10^{\circ}$ ano $(38,60 \%)$ nunca reprovou $(57,40 \%)$; distam a mais de $7 \mathrm{~km}$ da escola, demorando menos de 30 minutos a realizar o percurso entre casa e a escola ou vice-versa e deslocam-se em transportes públicos (50,50\%). O valor médio para a pontuação global do questionário reduzido de auto-regulação (SSRQ) foi de 89,83 (Dp=9,27), com um mínimo de 52 e um máximo de 145 e dispersão fraca $(\mathrm{CV}=10,32 \%)$. Os valores de simetria $(\mathrm{Sk} / \mathrm{erro}=1,436)$ e kurtose $(\mathrm{K} / \mathrm{erro}=26,841)$ revelaram uma distribuição simétrica e leptocúrtica. $\mathrm{Na}$ subescala "Estabelecimento de Objetivos", o valor médio obtido foi de 56,43 com um desvio padrão de 8,17. Os valores mínimos e máximos foram de 23 e 75 , respectivamente. A variável revelou uma dispersão fra$\mathrm{ca}(\mathrm{CV}=14,48 \%)$. 
A distribuição revelou-se assimétrica negativa ou enviesada à direita $(\mathrm{Sk} /$ erro $=-4,435)$ e mesocúrtica $(\mathrm{K} /$ erro=1,484).

A subescala "Controlo de Impulsos" apresentou um valor médio de 33,40 (Dp=8,60), com um valor mínimo de 14 e um máximo de 70. A dispersão revelou-se elevada apresentando um CV de 25,75\%. Os valores de simetria $(\mathrm{Sk} / \mathrm{erro}=3,333)$ e de Kurtose $(\mathrm{K} / \mathrm{erro}=1,643)$ indicaram uma distribuição assimétrica positiva ou enviesada à esquerda e mesocúrtica (Tabela 1).

Tabela 1 - Estatísticas relativas à pontuação global de auto-regulação e suas subescalas

\begin{tabular}{|l|l|l|l|l|l|l|l|l|l|}
\hline $\begin{array}{r}\text { Auto- } \\
\text { regulação } \\
\text { Variáveis }\end{array}$ & $\mathrm{N}$ & $\mathrm{X}$ & $\mathrm{Min}$ & $\mathrm{Max}$ & $\mathrm{Dp}$ & $\mathrm{CV} \%$ & $\begin{array}{l}\text { Sk/ } \\
\text { erro }\end{array}$ & K/erro & $\mathrm{K} / \mathrm{S}$ \\
\hline $\begin{array}{l}\text { Estab. de } \\
\text { Objetivos }\end{array}$ & 971 & 56,43 & 23 & 75 & 8,17 & 14,48 & $-4,435$ & 1,484 & 0,000 \\
\hline $\begin{array}{l}\text { Controlo } \\
\text { de Impul- } \\
\text { sos }\end{array}$ & 971 & 33,40 & 14 & 70 & 8,60 & 25,75 & 3,333 & 1,643 & 0,000 \\
\hline $\begin{array}{l}\text { SSRQ } \\
\text { (Total) }\end{array}$ & 971 & 89,83 & 52 & 145 & 9,27 & 10,32 & 1,436 & 26,841 & 0,000 \\
\hline
\end{tabular}

\section{Sexo vs Competências de Auto-regulação}

Os rapazes pontuam mais no controlo de impulsos e auto-regulação (global) e as raparigas no estabelecimento de objetivos, sem diferenças significativas (Tabela 2).

Tabela 2 - Teste $t$ de student relativo às competências de auto-regulação e suas subescalas em função do sexo

\begin{tabular}{|c|c|c|c|c|c|c|c|}
\hline Sexo & Mascu & & Femin & & Levene & t & \\
\hline Variáveis & $\mathrm{X}$ & $\mathrm{Dp}$ & $\mathrm{X}$ & Dp & $\mathrm{p}$ & $\tau$ & $\mathrm{p}$ \\
\hline Controlo de Impulsos & 33,86 & 8,59 & 32,92 & 8,60 & 0,676 & $-1,699$ & 0,090 \\
\hline $\begin{array}{l}\text { Estabelecimento de } \\
\text { objetivos }\end{array}$ & 56,25 & 8,56 & 56,62 & 7,75 & 0,111 & 0,697 & 0,486 \\
\hline Auto-regulação (total) & 90,11 & 9,96 & 89,54 & 8,51 & 0,097 & $-0,961$ & 0,337 \\
\hline
\end{tabular}

Observou-se que $41,30 \%$ dos estudantes apresentou reduzidas competências de auto-regulação, secundada pelos que revelaram elevadas competências de auto-regulação $31,10 \%$ e $27,60 \%$ revelaram razoáveis competências de auto-regulação, 40,00\% dos rapazes e $42,60 \%$ das raparigas revelaram reduzidas competências de auto-regulação sem diferenças estatisticamente significativas (Tabela 3 ).

Tabela 3 - Níveis de competências de auto-regulação em função do sexo

\begin{tabular}{|l|l|l|l|l|l|l|l|l|l|}
\hline \multicolumn{1}{|r|}{ Sexo } & \multicolumn{2}{|l|}{ Masculino (1) } & \multicolumn{2}{l|}{ Feminino (2) } & \multicolumn{2}{l|}{ Total } & \multicolumn{2}{l|}{ Residuais } \\
\hline SSRQ (Gr) & $\mathrm{N}$ & $\%$ & $\mathrm{~N}$ & $\%$ & $\mathrm{~N}$ & $\%$ & $(1)$ & $(2)$ \\
\hline Reduzidas & 197 & 40,00 & 204 & 42,60 & 401 & 41,30 & $-0,9$ & 0,9 \\
\hline Razoáveis & 131 & 26,50 & 137 & 28,70 & 268 & 27,60 & $-0,7$ & 0,7 \\
\hline Elevadas & 165 & 33,50 & 137 & 28,70 & 302 & 31,10 & 1,6 & $-1,6$ \\
\hline Total & 493 & 100,00 & 478 & 100,00 & 971 & 100,00 & & \\
\hline
\end{tabular}

\section{Idade vs Competências de Auto-regulação}

No controlo de impulsos são os estudantes com 16 anos ou menos que revelaram maiores competências de controlo de impulsos, sem diferenças estatisticamente significativas ( $F=1,611 ; p=0,200-n . s$.$) . Foram os estu-$ dantes com 17 anos que melhor estabeleciam objetivos, mas sem diferenças estatisticamente significativas $(\mathrm{F}=$ 0,603; $\mathrm{p}=0,547$-n.s.). Na pontuação global da escala são os estudantes com 16 anos que apresentam maiores índices de auto-regulação sem significância estatística $(\mathrm{F}=0,511 ; \mathrm{p}=0,600-$ n.s.) (Tabela 4).

Tabela 4 - Análise da variância entre as competências de auto-regulação, suas subescalas e grupos etários

\begin{tabular}{|l|l|l|l|l|l|l|l|l|}
\hline \multicolumn{1}{r|}{$\begin{array}{r}\text { Grupos } \\
\text { Etários }\end{array}$} & $\leq 16$ anos & \multicolumn{2}{|l|}{17 anos } & \multicolumn{2}{|l|}{$\geq 18$ anos } & \multirow{F}{*}{$\mathrm{F}$} & $\mathrm{p}$ \\
\hline Variáveis & $\mathrm{X}$ & $\mathrm{Dp}$ & $\mathrm{X}$ & $\mathrm{Dp}$ & $\mathrm{X}$ & $\mathrm{Dp}$ & & \\
\hline $\begin{array}{l}\text { Controlo de } \\
\text { Impulsos }\end{array}$ & 33,90 & 8,47 & 32,72 & 8,38 & 33,35 & 9,04 & 1,611 & 0,200 \\
\hline $\begin{array}{l}\text { Estab. de } \\
\text { objetivos }\end{array}$ & 56,28 & 8,20 & 56,87 & 7,86 & 56,19 & 8,47 & 0,603 & 0,547 \\
\hline $\begin{array}{l}\text { Auto-regula- } \\
\text { ção (total) }\end{array}$ & 90,17 & 9,14 & 89,59 & 8,97 & 89,54 & 9,84 & 0,511 & 0,600 \\
\hline
\end{tabular}

\section{DISCUSSÃO}

\section{Auto-regulação e Variáveis Sociodemográficas}

Ao estudar a auto-regulação em função do sexo, verificou-se que globalmente, os rapazes $(=90,11 ; \mathrm{Dp}=9,96)$ revelaram índices médios superiores aos das raparigas ( $=89,54 ; \mathrm{Dp}=8,51)$. Os resultados obtidos vão de encontro aos estudos de Carey, Neal \& Collins (2004), Neal \& Carey (2005), Hustad et al., (2009) e Castillo \& Dias (2007). Com efeito, no estudo de Castillo \& Dias (2007), o sexo masculino revela pontuações médias superiores às do sexo feminino. Também na subescala "estabelecimento de objetivos", as raparigas revelaram melhores pontuações médias comparativamente aos rapazes.

Relativamente à idade, os estudos de Carey et al., (2004), Neal \& Carey (2005), assim como os de Castillo \& Dias (2007 e 2009) não encontraram significâncias estatísticas. No nosso estudo, os resultados encontrados corroboram os resultados anteriormente mencionados pois, são os estudantes com idade igual ou inferior a 16 anos, que revelaram melhores índices de auto-regulação e melhor pontuaram na subescala "controlo de impulsos", apesar da ausência de significância estatística. No estabelecimento de objetivos, foram os estudantes da faixa etária dos 17 anos, os que melhores competências revelaram. 
Os estudantes residentes em meio rural apresentavam melhor capacidade de controlo de impulsos, com diferenças estatisticamente significativas $(p=0,044)$ e melhores índices de auto-regulação, sem relevância estatística ( $\mathrm{p}>0,050-\mathrm{n} . \mathrm{s}$.). Os residentes em meio urbano pontuam melhor no estabelecer de objetivos, embora sem significado estatístico ( $\mathrm{p}=0,160-\mathrm{n} . \mathrm{s}$.). Relativamente à influência da área de residência na auto-regulação não encontrámos estudos que servissem de apoio ou refutassem os dados por nós obtidos.

$\mathrm{O}$ rendimento médio do agregado familiar não se revelou influenciador dos índices de auto-regulação globais dos estudantes ( $t=1,772 ; \mathrm{p}=0,077$-n.s.), apesar dos estudantes de agregado familiar baixo ou médio/baixo obterem melhores índices médios de auto-regulação. Estes dados apoiam os de Neal \& Carey (2005).

\section{Auto-regulação e Envolvimento Face ao Álcool}

A maioria dos estudantes são bebedores habituais sem problemas $(75,30 \%)$, dados estes que estão em consonância com os obtidos por Fonte \& Alves (1999). Rapazes $(72,40 \%)$ e raparigas $(78,20 \%)$ são bebedores habituais sem problemas.

Para Reis et al., (2011) 66,3\% dos rapazes e 70,2\% das raparigas apresentam consumo de risco (o equivalente à categoria de bebedores habituais sem problemas na escala de envolvimento com o álcool para adolescentes). Vilela, Macho e Almeida (2011) encontraram 68\% de bebedores habituais sem problemas.

Verificámos um envolvimento com o álcool superior em rapazes $(t=-4,493 ; p=0,000)$ com significância estatística. Reis et al., (2011) também constatou a existência de uma relação estatisticamente significativa entre os sexos, sendo o sexo masculino os que revelaram um maior envolvimento com o álcool $(\mathrm{p}=0,032)$. No que diz respeito à idade, encontrámos significância estatística entre os grupos estudados, sendo os alunos com 18 anos os que apresentam maior envolvimento com o ál$\operatorname{cool}(\mathrm{F}=14,066 ; \mathrm{p}=0,000)$. Os alunos com idades inferiores ou iguais a 16 anos são os que menor envolvimento têm com o álcool. Vilela, Macho \& Mendes (2011) assim como Reis et al., (2011) verificaram igualmente a existência de diferenças estatisticamente significativas entre o envolvimento com o álcool e a idade $(\mathrm{p}<0,001)$, concluindo que o envolvimento com o álcool aumenta com a idade dos adolescentes.

Os residentes na área rural mostraram um maior envolvimento com o álcool sem diferenças encontradas estatisticamente significativas ( $\mathrm{p}=0,855$-n.s.).
Estes dados contrariam os de Mendes e Lopes (2007). Os estudantes do $12^{\circ}$ ano possuem um maior envolvimento com o álcool, com diferenças estatisticamente significativas $(\mathrm{F}=5,422 ; \mathrm{p}=0,005)$, para os estudantes do $10^{\circ}$ e $11^{\circ}$ anos. Dados concordantes com os de Vilela et al., (2011). Relativamente ao insucesso escolar verificámos que os alunos com reprovações apresentaram um maior envolvimento com o álcool $(\mathrm{t}=-4,087 ; \mathrm{p}=$ $0,000)$, com significância estatística. O envolvimento com o álcool (nota global) surge nos resultados obtidos, como uma variável preditora da auto-regulação (índice global), com uma correlação negativa muito baixa $(\mathrm{r}=-0,056 ; \mathrm{p}=0,040)$. Assim, melhores índices de auto-regulação encontram-se associados a um menor envolvimento com o álcool. Também os estudos de Brown et al., (1999) e de Aubrey, Brown \& Miller (1994) obtêm resultados próximos dos nossos. À semelhança do estudo de Neal \& Carey (2005), também no nosso estudo, as consequências relacionadas com o consumo se demonstraram preditivas dos índices globais de auto-regulação. De facto, a dimensão relativa aos efeitos, consequências e sentimentos face ao beber da escala de envolvimento com o álcool em adolescentes surgiu como uma variável preditiva da auto-regulação, estabelecendo uma correlação positiva (muito baixa) ( $\mathrm{r}=$ $0,128 ; \mathrm{p}=0,000)$.

Quanto ao controlo de impulsos associaram-se positivamente os efeitos, consequências e sentimentos face ao beber $(r=0,162 ; p=0,000)$ da subescala do envolvimento face ao álcool, com uma correlação muito baixa, ou seja, a maiores efeitos, consequências e sentimentos face ao álcool correspondem melhores capacidades de controlo de impulsos.

Os hábitos de consumo correlacionaram-se inversamente com o estabelecimento de objetivos $(r=-0,067$; $\mathrm{p}=0,019)$, isto é, menores hábitos de consumo de álcool por parte dos adolescentes surgem, neste estudo, associados a melhores capacidades de estabelecimento de objetivos. Estes resultados estão em consonância com os resultados de Castillo \& Dias (2007 e 2009).

\section{Auto-regulação e Expectativas Face ao Álcool}

Os estudantes apresentaram uma pontuação global média de $114,56(\mathrm{Dp}=35,11)$ na escala global de expectativas face ao álcool. $\mathrm{O}$ valor médio obtido no que concerne às expectativas positivas foi de 59,23 (Dp= $19,29)$ e de $55,32(\mathrm{Dp}=19,82)$ relativamente às expectativas negativas. Pillati et al., (2010), na validação da sua escala obtiveram valores médios inferiores aos obtidos no nosso estudo. 
$\mathrm{Na}$ pontuação global da escala, a maioria dos estudantes apresentam elevadas expectativas face ao álcool $(45,10 \%)$, sendo os rapazes os que apresentam valores superiores (50,30\%).Estes dados estão em consonância com os obtidos por Pillati et al., (2010).

Ao nível da idade, verificámos que a pontuação média relativa às expectativas positivas aumentou com a idade. Quanto às expectativas negativas, registámos o inverso, ou seja, uma diminuição nas pontuações médias de expectativas negativas com o aumento da idade. Estes dados são corroborados por Pillati et al., (2010). Apenas constatámos significância estatística na subescala das expectativas negativas entre os alunos com idades inferiores ou iguais a 16 anos e os alunos com 18 anos ou mais $(F=5,090 ; p=0,007)$.

Relativamente ao envolvimento com o álcool, constatámos a existência de correlação baixa e positiva ( $\mathrm{r}=$ 0,303; $\mathrm{p}<0,010$ ) com as expectativas negativas, ou seja, índices mais elevados no nível de expectativas positivas face ao álcool, maior o envolvimento com o álcool. Já as expectativas negativas não se correlacionaram significativamente com o envolvimento com o álcool $(\mathrm{r}=$ 0,006; p> 0,050-n.s.).

Nesta investigação, maiores expectativas face ao álcool (globais) associaram-se a maiores índices de auto-regulação. Embora a correlação seja baixa e positiva ( $\mathrm{r}=$ $0,179 ; \mathrm{p}=0,000)$. Também as expectativas face ao álcool (globais) se revelaram preditivas do controlo de impulsos, ao estabelecer uma associação positiva, embora fraca $(\mathrm{r}=0,298 ; \mathrm{p}=0,000)$.

\section{CONCLUSÃO}

O comportamento auto-regulado associado à saúde individual consiste em delinear objetivos a longo prazo implicando o controlo dos impulsos mais imediatos. Os estilos de vida, tais como o consumo de álcool, são susceptíveis de serem auto-regulados.

A partir dos resultados, concluímos que, as competências de auto-regulação, os efeitos, consequências e sentimentos face ao beber e as expectativas globais face ao álcool surgem como preditoras do controlo de impulsos. Os hábitos de consumo surgem como preditores do estabelecimento de objetivos e os efeitos, consequências e sentimentos face ao beber, o envolvimento com o álcool (global) e as expectativas globais face ao álcool são preditoras da auto-regulação (total). São os rapazes que revelam maior envolvimento com o álcool, sendo na sua maioria, bebedores habituais sem problemas.
Possuem um maior envolvimento com o álcool, estudantes com idade igual ou superior a 18 anos, com maiores valores monetários para gastos pessoais, no $12^{\circ}$ ano, numa escola Profissional com história de reprovações prévias e que referem não gostar da escola. Quanto às expectativas face ao álcool, 45,10\% revela elevadas expectativas, revelando ainda um maior nível de expectativas globais os rapazes, do $10^{\circ}$ ano, da escola profissional, que não gostam da escola e que são bebedores habituais com problemas.

\section{IMPLICAÇÕES PARA A PRÁTICA CLÍNICA}

Parece-nos importante planear, implementar e avaliar intervenções que melhorem o clima relacional nas escolas, nomeadamente ao nível das variáveis de contexto psicológico (auto-estima, autoconceito e comportamento auto-regulado). A escola é um espaço privilegiado para trabalhar no âmbito da educação para a saúde, sendo facilitadora da mudança de atitudes e de comportamentos face ao consumo de álcool, devendo esta assumir-se como promotora de estilos de vida saudáveis.

\section{REFERÊNCIAS BIBLIOGRÁFICAS}

Aubrey, L. L., Brown, J. M., \& Miller, W. R., (1994). Psychometric properties of a self-regulation questionnaire. Alcoholism: Clinical \& Experimental Research, 18, 429.

Brown, J. M., Miller, W. R., \& Lawendowski, L. A., (1999). The Self-Regulation Questionnaire. Em VandeCreek, L., \& Jackson, T. L., (Eds.), Innovations in Clinical Practice: A sourcebook, 17, 281-292.

Carey, K.B., Neal, D. J., \& Collins, S.E., (2004). A psychometric analysis of the self-regulation questionnaire. Addictive Behaviours - Journal, 29(2), 253-260.

Castillo, J. A. G., \& Dias, P. C., (2009). Auto-regulação, resiliência e consumo de substâncias na adolescência: contributos da adaptação do questionário reduzido de auto-regulação. Psicologia, Saúde \& Doenças, 10(2), 205-216.

Castillo, J. G., \& Dias, P., (2007). Análisis relacional entre los factores de protección, resiliencia, autorregulación y consumo de drogas. Salud y Drogas, 7(2), 309-332. 
Fonte, A., \& Alves, A., (1999). Uso da Escala de Envolvimento com o Álcool para Adolescentes (AAIS). Avaliação das Características Psicométricas. Alcoologia, Revista da Sociedade Portuguesa de Alcoologia, 7(4). Acedido em http://anibalfonte.home.sapo.pt/ AAIS.html

Hull, J. G., \& Slone, L. B., (2004). Alcohol and Self-Regulation. In R. F. Baumeister, \& K. D. Vohs (Eds.), Handbook of Self-Regulation: Research, Theory, and Applicatins, 466-491. New York, United States of America: Guilford Publications.

Hustad, J., Carey, K., Carey, M., \& Maisto, S., (2009). Self-Regulation, Alcohol consumption, and Consequences in College Student Heavy Drinkers: A Simultaneous Latent Growth Analysis. Journal of Studies on Alcohol and Drugs, 70(3), 373-382.

Mendes, V., \& Lopes, P., (2007). Hábitos de consumo de álcool em adolescentes. Revista Toxicodependências, 13(2), 25-40.

Neal, D. J., \& Carey, K. B., (2005). A Follow-Up Psychometric Analysis of the Self-Regulation Questionnaire. Psycholy Addictive Behaviour, 19(4), 414-422.

Pilatti, A., Godoy, J. C., \& Brussino, S. A., (2010). Construcción y valoración psicométrica del Cuestionario de Expectativas hacia el Alcohol para Adolescentes de Argentina (CEA-A). Anales de Psicología, 26(2), 288-301.
Reis, A., Barros, J., Fonseca, C., Parreira, L., Gomes, M., Figueiredo, I., \& Matapa, S., (2011). Prevalência da Ingestão de Álcool nos Adolescentes - Estudo PINGA. Revista Portuguesa de Clínica Geral, 27(4), 338-346.

Ridder, D., \& Wit, J. (Eds.) (2006). Self-Regulation in Health Behavior. Chichester, United Kingdom: Wiley.

Sousa, F. C., Abraão, A. M., Morgado, A., Conboy, J., Oliveira, M. D., \& Pires, D., (2008). O consumo de bebidas alcoólicas na população escolar juvenil ( $\left.1^{\text {a }} \mathrm{ed}\right)$. Loulé, Portugal: GAIM.

Strauch, E. S., Pinheiro, R. T., Silva, R. A., \& Horta, B. L., (2009). Uso de álcool por adolescentes: estudo de base populacional. Revista Saúde Pública, 43(4), 647-655.

Tavares, T., Bonito, J., \& Oliveira, M. M. (2011). Consumo de álcool entre os alunos do 9. ${ }^{\circ}$ ano de escolaridade do distrito de Beja: Fatores caracterizantes. In I Congresso Nacional de Comportamentos de Saúde InfantoJuvenis, Consumo de substâncias psicoactivas: lícitas e ilícitas (pp. 57-73). Livro de resumos, Viseu.

Vilela, L., Macho, P., \& Almeida, G., (2011). Consumo de álcool em adolescentes e psicopatologia associada. Toxicodependências, 17(1), 43-52.

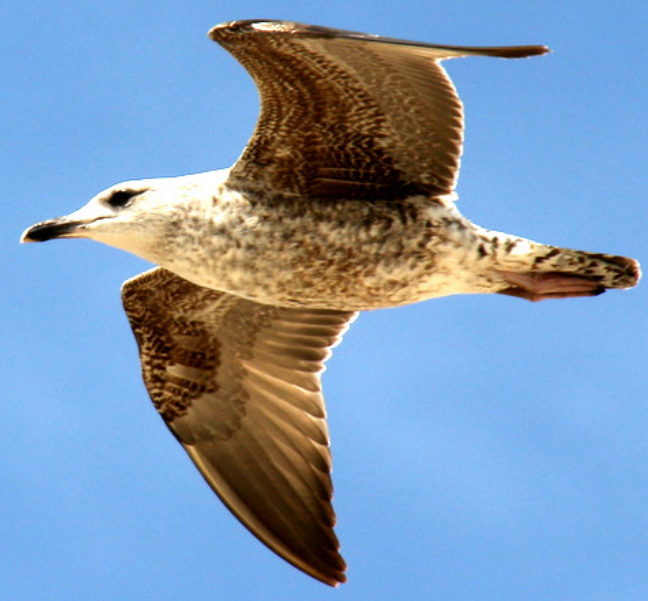

